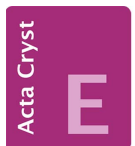
COMMUNICATIONS

ISSN 2056-9890

\section{Crystal structure of the pyridine-diiodine (1/1) adduct}

\author{
Matti Tuikka and Matti Haukka* \\ University of Jyvaskyla, Department of Chemistry, P.O. Box 35, FI-40014 University
} of Jyvaskyla, Finland. *Correspondence e-mail: matti.o.haukka@jyu.fi

Received 17 April 2015; accepted 1 June 2015

Edited by C. Rizzoli, Universita degli Studi di Parma, Italy

In the title adduct, $\mathrm{C}_{5} \mathrm{H}_{5} \mathrm{~N} \cdot \mathrm{I}_{2}$, the $\mathrm{N}-\mathrm{I}$ distance $[2.424(8) \AA]$ is remarkably shorter than the sum of the van der Waals radii. The line through the I atoms forms an angle of $78.39(16)^{\circ}$ with the normal to the pyridine ring.

Keywords: pyridine; diiodine; halogen bonding; crystal structure.

CCDC reference: 1404151

\section{Related literature}

For the structure of the pyridine- $\mathrm{I}_{2}$ 1:2 adduct, see: Hassel \& Hope (1961). For the crystal structures of pyridine with $\mathrm{ICl}$ and IBr, see: Rømming (1972); Dahl et al. (1967). For van der Walls radii, see: Bondi (1964). For the I-I distance of iodine, see: Buontempo et al. (1997). For $\mathrm{I}-\mathrm{I}^{\cdots} \mathrm{N}$ angles in halogen bonding, see: Desiraju et al. (2013).

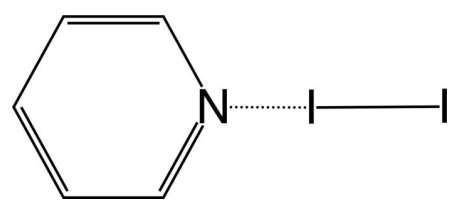

\section{Experimental}

\subsection{Crystal data}

$\mathrm{C}_{5} \mathrm{H}_{5} \mathrm{~N} \cdot \mathrm{I}_{2}$

$M_{r}=332.90$

Monoclinic, $P 2 / C$

$a=9.2432$ (6) $\AA$

$b=4.3392$ (2) $\AA$

$$
\begin{aligned}
& c=20.1953(13) \AA \\
& \beta=98.468(3)^{\circ} \\
& V=801.16(8) \AA^{3} \\
& Z=4 \\
& \text { Mo } K \alpha \text { radiation }
\end{aligned}
$$

$\mu=7.76 \mathrm{~mm}^{-1}$

$T=120 \mathrm{~K}$

\subsection{Data collection}

Bruker KAPPA APEX II CCD diffractometer

Absorption correction: numerical (SADABS; Bruker,2012)

$T_{\min }=0.574, T_{\max }=0.902$

\subsection{Refinement}

$R\left[F^{2}>2 \sigma\left(F^{2}\right)\right]=0.049$

$w R\left(F^{2}\right)=0.091$

$S=1.07$

1853 reflections

73 parameters

$\mathrm{H}$-atom parameters constrained

$\Delta \rho_{\max }=1.11{\mathrm{e} \AA^{-3}}^{-3}$

$\Delta \rho_{\min }=-1.26 \mathrm{e}^{-3}$

Data collection: Collect (Nonius, 2000); cell refinement: DENZO/ SCALEPACK (Otwinowski \& Minor, 1997); data reduction: DENZO/SCALEPACK; program(s) used to solve structure: SUPERFLIP (Palatinus \& Chapuis, 2007; Palatinus \& van der Lee, 2008; Palatinus et al., 2012); program(s) used to refine structure: SHELXL97 (Sheldrick, 2008); molecular graphics: OLEX2 (Dolomanov et al., 2009); software used to prepare material for publication: OLEX2.

\title{
Acknowledgements
}

Financial support provided by the Academy of Finland (project No. 129171) is gratefully acknowledged.

Supporting information for this paper is available from the IUCr electronic archives (Reference: RZ5157).

\section{References}

Bondi, A. (1964). J. Phys. Chem. 68, 441-451.

Bruker (2012). SADABS. Bruker AXS Inc., Madison, Wisconsin, USA.

Buontempo, U., DiCicco, A., Filipponi, A., Nardone, M. \& Postorino, P. (1997). J. Chem. Phys. 107, 5720-5726.

Dahl, T., Hassel, O. \& Sky, K. (1967). Acta Chem. Scand. 21, 592-593.

Desiraju, G. R., Ho, P. S., Kloo, L., Legon, A. C., Marquardt, R., Metrangolo, P., Politzer, P., Resnati, G. \& Rissanen, K. (2013). Pure Appl. Chem. 85, 1711-1713.

Dolomanov, O. V., Bourhis, L. J., Gildea, R. J., Howard, J. A. K. \& Puschmann, H. (2009). J. Appl. Cryst. 42, 339-341.

Hassel, O. \& Hope, H. (1961). Acta Chem. Scand. 15, 407-416.

Nonius (2000). COLLECT. Nonius BV, Delft, The Netherlands.

Otwinowski, Z. \& Minor, W. (1997). Methods in Enzymology, Vol. 276, Macromolecular Crystallography, Part A, edited by C. W. Carter Jr \& R. M Sweet, pp. 307-326. New York: Academic Press.

Palatinus, L. \& Chapuis, G. (2007). J. Appl. Cryst. 40, 786-790.

Palatinus, L., Prathapa, S. J. \& van Smaalen, S. (2012). J. Appl. Cryst. 45, 575580.

Palatinus, L. \& van der Lee, A. (2008). J. Appl. Cryst. 41, 975-984.

Rømming, C. (1972). Acta Chem. Scand. 26, 1555-1560.

Sheldrick, G. M. (2008). Acta Cryst. A64, 112-122. 


\section{supporting information}

Acta Cryst. (2015). E71, o463 [doi:10.1107/S2056989015010518]

\section{Crystal structure of the pyridine-diiodine (1/1) adduct}

\section{Matti Tuikka and Matti Haukka}

\section{S1. Comment}

Diiodine is capable to act as halogen bond donor and form stable halogen bonds with Lewis bases, such as pyridine, due to the strong charge transfer. In the case of the pyridine- $\mathrm{I}_{2}$ 1:2 adduct (Hassel \& Hope, 1961), the interaction eventually results in the heterolytic cleavage of $\mathrm{I}_{2}$ and formaton of $\left[\mathrm{py}_{2} \mathrm{I}\right]^{+} \mathrm{I}_{3}{ }^{-}$ion pairs. Although the crystal structures involving pyridine and interhalogens ICl and IBr are known (Rømming, 1972; Dahl et al., 1967), the title pyI $\mathrm{I}_{2} 1: 1$ adduct has not been reported earlier. The N1-I1 distance in $\mathrm{pyI}_{2}(2.425(8) \AA)$ is remarkably shorter than the sum of the van der Walls radii of iodine and nitrogen (3.53 $\AA$; Bondi, 1964). The I-I distance (2.8043 (9) $\AA$ ) is significantly longer than that

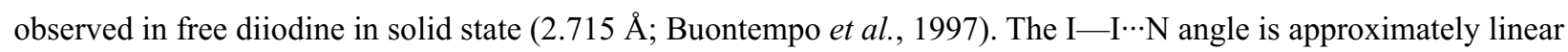
$\left(176.44(18)^{\circ}\right)$ as expected in halogen bonds (Desiraju et al., 2013).

\section{S2. Experimental}

The title compound was synthesized by dissolving iodine $(200 \mathrm{mg})$ in ethanol $(5 \mathrm{ml})$ and adding pyridine $(1 \mathrm{ml})$ into this solution. The solution was left to evaporate unde ambient conditions and after a couple of days light yellow crystals were formed.

\section{S3. Refinement}

All $\mathrm{H}$ atoms were positioned geometrically and refined using a riding model with $\mathrm{C}-\mathrm{H}=0.95 \AA$ and with $U_{\text {iso }}(\mathrm{H})=1.2$ $U_{\text {eq }}(\mathrm{C})$.

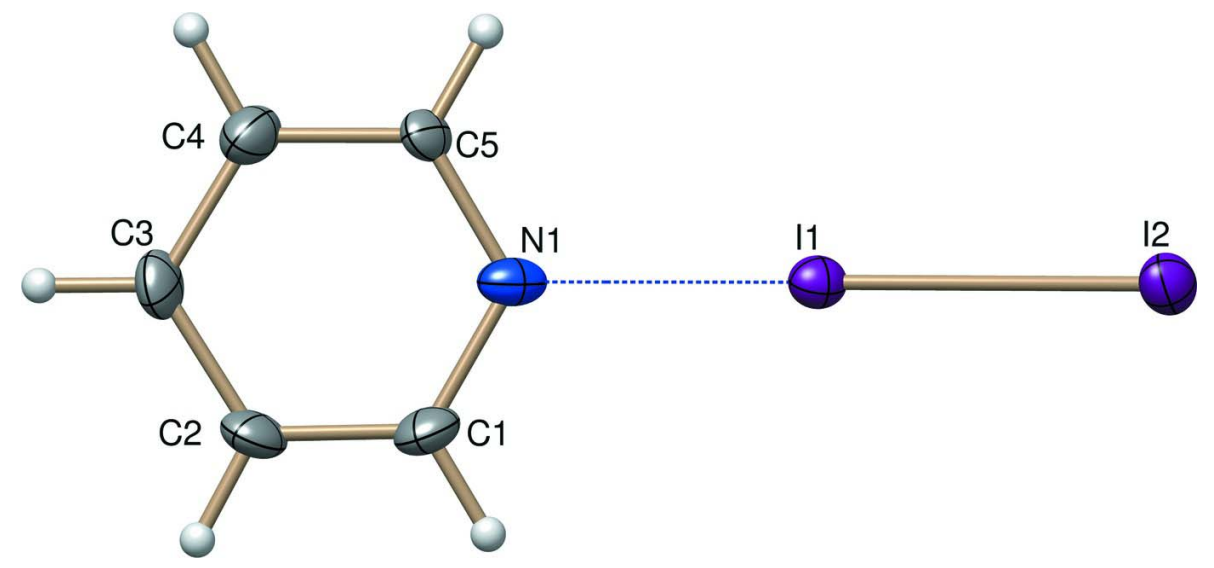

\section{Figure 1}

The molecular structure of the title compound, with 50\% probability displacement ellipsoids for non- $\mathrm{H}$ atoms. 


\section{Pyridine-diiodine (1/1)}

Crystal data

$\mathrm{C}_{5} \mathrm{H}_{5} \mathrm{~N} \cdot \mathrm{I}_{2}$

$M_{r}=332.90$

Monoclinic, $P 2 / \mathrm{c}$

$a=9.2432(6) \AA$

$b=4.3392(2) \AA$

$c=20.1953(13) \AA$

$\beta=98.468(3)^{\circ}$

$V=801.16(8) \AA^{3}$

$Z=4$

Data collection

Bruker KAPPA APEX II CCD diffractometer

Radiation source: fine-focus sealed tube

Curved graphite crystal monochromator

Detector resolution: 16 pixels $\mathrm{mm}^{-1}$

$\varphi$ scans and $\omega$ scans with $\kappa$ offset

Absorption correction: numerical

(SADABS; Bruker,2012)

$T_{\min }=0.574, T_{\max }=0.902$

Refinement

Refinement on $F^{2}$

Least-squares matrix: full

$R\left[F^{2}>2 \sigma\left(F^{2}\right)\right]=0.049$

$w R\left(F^{2}\right)=0.091$

$S=1.07$

1853 reflections

73 parameters

0 restraints

Primary atom site location: iterative

Special details

Geometry. All e.s.d.'s (except the e.s.d. in the dihedral angle between two 1.s. planes) are estimated using the full covariance matrix. The cell e.s.d.'s are taken into account individually in the estimation of e.s.d.'s in distances, angles and torsion angles; correlations between e.s.d.'s in cell parameters are only used when they are defined by crystal symmetry. An approximate (isotropic) treatment of cell e.s.d.'s is used for estimating e.s.d.'s involving 1.s. planes.

Fractional atomic coordinates and isotropic or equivalent isotropic displacement parameters $\left(\AA^{2}\right)$

\begin{tabular}{lllll}
\hline & $x$ & $y$ & $z$ & $U_{\text {iso }} * / U_{\text {eq }}$ \\
\hline I1 & $0.27234(6)$ & $0.59684(13)$ & $0.54587(3)$ & $0.02083(16)$ \\
I2 & $0.32645(6)$ & $0.35101(14)$ & $0.67558(3)$ & $0.02480(18)$ \\
N1 & $0.2243(7)$ & $0.8407(18)$ & $0.4368(4)$ & $0.0243(18)$ \\
C5 & $0.3349(9)$ & $0.933(2)$ & $0.4053(4)$ & $0.0207(19)$ \\
H5 & 0.4323 & 0.8743 & 0.4223 & $0.025^{*}$ \\
C3 & $0.1668(10)$ & $1.198(2)$ & $0.3214(5)$ & $0.028(2)$ \\
H3 & 0.1471 & 1.3178 & 0.2818 & $0.033^{*}$ \\
C1 & $0.0849(10)$ & $0.921(2)$ & $0.4121(5)$ & $0.030(2)$ \\
H1 & 0.0069 & 0.8529 & 0.4342 & $0.035^{*}$
\end{tabular}

$F(000)=592$

$D_{\mathrm{x}}=2.760 \mathrm{Mg} \mathrm{m}^{-3}$

Mo $K \alpha$ radiation, $\lambda=0.71073 \AA$

Cell parameters from 1865 reflections

$\theta=1.0-27.5^{\circ}$

$\mu=7.76 \mathrm{~mm}^{-1}$

$T=120 \mathrm{~K}$

Plate, clear light yellow

$0.09 \times 0.07 \times 0.02 \mathrm{~mm}$

6585 measured reflections

1853 independent reflections

1437 reflections with $I>2 \sigma(I)$

$R_{\text {int }}=0.062$

$\theta_{\text {max }}=27.6^{\circ}, \theta_{\min }=2.2^{\circ}$

$k=-5 \rightarrow 5$

$l=-26 \rightarrow 25$

Hydrogen site location: inferred from neighbouring sites

$\mathrm{H}$-atom parameters constrained

$w=1 /\left[\sigma^{2}\left(F_{\mathrm{o}}{ }^{2}\right)+(0.0153 P)^{2}+9.3396 P\right]$

where $P=\left(F_{\mathrm{o}}{ }^{2}+2 F_{\mathrm{c}}{ }^{2}\right) / 3$

$(\Delta / \sigma)_{\max }=0.001$

$\Delta \rho_{\max }=1.11 \mathrm{e} \AA^{-3}$

$\Delta \rho_{\min }=-1.26 \mathrm{e}^{-3}$ $h=-11 \rightarrow 11$ 


\begin{tabular}{lllll}
$\mathrm{C} 2$ & $0.0549(10)$ & $1.098(2)$ & $0.3558(5)$ & $0.030(2)$ \\
$\mathrm{H} 2$ & -0.0434 & 1.1533 & 0.3399 & $0.036^{*}$ \\
$\mathrm{C} 4$ & $0.3076(10)$ & $1.112(2)$ & $0.3480(5)$ & $0.030(2)$ \\
$\mathrm{H} 4$ & 0.3872 & 1.1783 & 0.3266 & $0.036^{*}$ \\
\hline
\end{tabular}

Atomic displacement parameters $\left(\AA^{2}\right)$

\begin{tabular}{lllllll}
\hline & $U^{11}$ & $U^{22}$ & $U^{33}$ & $U^{12}$ & $U^{13}$ & $U^{23}$ \\
\hline I1 & $0.0189(3)$ & $0.0224(3)$ & $0.0212(3)$ & $-0.0010(2)$ & $0.0027(2)$ & $-0.0016(3)$ \\
I2 & $0.0253(3)$ & $0.0267(3)$ & $0.0222(4)$ & $0.0029(2)$ & $0.0031(3)$ & $0.0011(3)$ \\
N1 & $0.016(4)$ & $0.032(4)$ & $0.024(4)$ & $-0.003(3)$ & $0.004(3)$ & $-0.007(4)$ \\
C5 & $0.017(4)$ & $0.031(5)$ & $0.013(5)$ & $-0.001(4)$ & $-0.001(3)$ & $0.000(4)$ \\
C3 & $0.031(5)$ & $0.035(6)$ & $0.014(5)$ & $-0.002(4)$ & $-0.003(4)$ & $0.004(4)$ \\
C1 & $0.019(5)$ & $0.039(6)$ & $0.031(6)$ & $-0.009(4)$ & $0.007(4)$ & $0.005(5)$ \\
C2 & $0.014(4)$ & $0.049(7)$ & $0.026(6)$ & $-0.002(4)$ & $-0.003(4)$ & $0.002(5)$ \\
C4 & $0.025(5)$ & $0.042(6)$ & $0.022(6)$ & $-0.006(4)$ & $0.005(4)$ & $0.003(5)$ \\
\hline
\end{tabular}

Geometric parameters $\left(\AA,{ }^{\circ}\right)$

\begin{tabular}{llll}
\hline $\mathrm{I} 1-\mathrm{I} 2$ & $2.8043(9)$ & $\mathrm{C} 5-\mathrm{C} 4$ & $1.388(13)$ \\
$\mathrm{I} 1-\mathrm{N} 1$ & $2.425(8)$ & $\mathrm{C} 3-\mathrm{C} 2$ & $1.397(12)$ \\
$\mathrm{N} 1-\mathrm{C} 5$ & $1.342(10)$ & $\mathrm{C} 3-\mathrm{C} 4$ & $1.383(13)$ \\
$\mathrm{N} 1-\mathrm{C} 1$ & $1.357(12)$ & $\mathrm{C} 1-\mathrm{C} 2$ & $1.364(14)$ \\
& & & \\
$\mathrm{N} 1-\mathrm{I} 1-\mathrm{I} 2$ & $176.44(18)$ & $\mathrm{C} 4-\mathrm{C} 3-\mathrm{C} 2$ & $116.6(9)$ \\
$\mathrm{C} 5-\mathrm{N} 1-\mathrm{I} 1$ & $120.7(6)$ & $\mathrm{N} 1-\mathrm{C} 1-\mathrm{C} 2$ & $121.1(8)$ \\
$\mathrm{C} 5-\mathrm{N} 1-\mathrm{C} 1$ & $119.8(8)$ & $\mathrm{C} 1-\mathrm{C} 2-\mathrm{C} 3$ & $120.9(9)$ \\
$\mathrm{C} 1-\mathrm{N} 1-\mathrm{I} 1$ & $118.9(6)$ & $\mathrm{C} 3-\mathrm{C} 4-\mathrm{C} 5$ & \\
$\mathrm{~N} 1-\mathrm{C} 5-\mathrm{C} 4$ & $120.3(8)$ & & \\
& & & $-0.8(15)$ \\
$\mathrm{I} 1-\mathrm{N} 1-\mathrm{C} 5-\mathrm{C} 4$ & $-170.3(7)$ & $\mathrm{C} 5-\mathrm{N} 1-\mathrm{C} 1-\mathrm{C} 2$ & $0.8(14)$ \\
$\mathrm{I} 1-\mathrm{N} 1-\mathrm{C} 1-\mathrm{C} 2$ & $170.4(8)$ & $\mathrm{C} 1-\mathrm{N} 1-\mathrm{C} 5-\mathrm{C} 4$ & $1.0(15)$ \\
$\mathrm{N} 1-\mathrm{C} 5-\mathrm{C} 4-\mathrm{C} 3$ & $-0.9(15)$ & $\mathrm{C} 2-\mathrm{C} 3-\mathrm{C} 4-\mathrm{C} 5$ & $-1.0(15)$ \\
$\mathrm{N} 1-\mathrm{C} 1-\mathrm{C} 2-\mathrm{C} 3$ & $1.0(16)$ & $\mathrm{C} 4-\mathrm{C} 3-\mathrm{C} 2-\mathrm{C} 1$ & \\
& & &
\end{tabular}

